\title{
Melflufen-A Novel Agent in the Treatment of Relapsed/Refractory Multiple Myeloma
}

\author{
An Expert Interview with Paul G Richardson
}

Jerome Lipper Multiple Myeloma Center, Department of Medical Oncology, Dana-Farber Cancer Institute, Harvard Medical School, Boston, MA, USA

DOI: https://doi.org/10.17925/OHR.2020.16.1.12

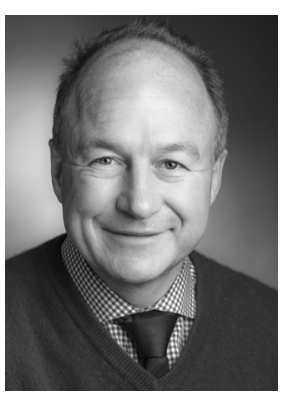

Paul G Richardson

Dr. Paul Richardson joined the Jerome Lipper Multiple Myeloma Center in 1999 after a visiting fellowship, and has served as faculty in cancer pharmacology at Dana-Farber Cancer Institute (DFCI) since 1994. He was appointed Clinical Director in 2001. His major clinical research has been focused on the development of novel agents for the treatment of multiple myeloma and combination strategies designed to improve efficacy, with one example being the landmark Intergroup Francophone Myelome (IFM)/DFCl clinical trial in newly diagnosed patients eligible for stem cell transplant treated with the combination of lenalidomide, bortezomib, and dexamethasone (RVD). He has authored or co-authored over 720 articles in peer-reviewed journals. In addition to holding positions on the editorial boards of leading journals, he has been Chairman of the Multiple Myeloma Research Consortium and the Clinical Trials Core, a member of the American Society of Clinical Oncology (ASCO) Hematologic Malignancies Subcommittee and ASCO Internet Cancer Information Committee, and has currently been Chair of the Alliance Myeloma Committee since 2011. He has achieved many honors and awards throughout his distinguished career, to name a few: the prestigious Warren Alpert Foundation Prize in recognition of the successful therapeutic targeting of the ubiquitin-proteasome pathway in 2012, one of Thomson Reuters Science Watch top 19 investigators at DFCI for the most highly cited research in 2016, the International Myeloma Foundation's Robert A Kyle Lifetime Achievement Award in 2017, and the Morse Research Award at DFCl in 2019 for outstanding clinical research.

\section{Keywords}

Multiple myeloma, melflufen, dexamethasone, relapsed/refractory

Disclosures: Paul G Richardson reports receiving grants from BMS; grants and/or honoraria, as an advisory committee member, from Celgene, Karyopharm, Oncopeptides, and Takeda; and honoraria, as an advisory committee member, from Janssen, Sanofi, and SecuraBio, all outside the submitted work.

Acknowledgment: Medical writing assistance was provided by Katrina Mountfort of Touch Medical Media, and supported by Touch Medical Media.

Review Process: This is an expert interview and, as such, has not undergone the journal's standard peer review process.

Compliance with Ethics: This is an expert interview and does not report on new clinical data, or any studies with human or animal subjects performed by the author.

Authorship: The named author meets the International Committee of Medical Journal Editors (ICMJE) criteria for authorship of this manuscript, takes responsibility for the integrity of the work as a whole, and has given final approval to the version to be published.

Access: This article is freely accessible at touchONCOLOGY.com (c) Touch Medical Media 2020.

Received: April 22, 2020

Published Online: July 30, 2020

Citation: Oncology \& Hematology Review (US). 2020; 16(1):12-4

Corresponding Author: Paul G Richardson, Harvard Medical School, Jerome Lipper Multiple Myeloma Center, Department of Medical Oncology, Dana-Farber Cancer Institute, Boston, MA 02215, USA. E: paul_richardson@dfci.harvard.edu

Support: No funding was received in the publication of this article.
$\mathrm{M}$ ultiple myeloma (MM) is the second most common hematological malignancy after non-Hodgkin's lymphoma. ${ }^{1}$ Although recent decades have seen considerable advances and improvements in clinical outcomes for patients with $\mathrm{MM}_{1}{ }^{2} \mathrm{MM}$ remains incurable, with a high disease burden. ${ }^{3}$ Clonal evolution and genomic instability within myeloma clones inevitably lead to disease progression and treatment resistance. ${ }^{4}$ An increasing number of patients are becoming refractory to multiple treatments; therefore, there is a need for new drugs with novel mechanisms of action.

Melflufen (melphalan flufenamide) is a novel peptide-drug conjugate that has shown efficacy in relapsed/refractory $\mathrm{MM}$. In an expert interview, Dr Richardson discusses the recently published O-12-M1 phase I/II study, which investigated the efficacy and safety of melflufen in the setting of triple-class refractory patients, i.e., those who have progressed after proteasome inhibitors, immune modulators, and antibodies.

\section{Q. What are the most important unmet needs in the treatment of multiple myeloma?}

There has been tremendous progress in the past 20 years with the advent of novel therapies, which initially focused on proteasome inhibition and immune modulation, and then the introduction of monoclonal antibodies. However, despite the success of these platforms, the disease remains incurable, and resistance and relapses are frequent, with the disease ultimately becoming refractory in each individual patient over time. Therefore, we are continuously looking for novel mechanisms of action that target fundamentally different aspects of tumor biology. In addition to B-cell maturation antigen (BCMA)-targeting strategies, including antibodies, but also excitingly chimeric antigen receptor T-cell (CAR-T) therapy, ${ }^{6}$ we have several small molecule targets including the selective inhibition of nuclear export proteins (SINE), the best example of which is selinexor, and the inhibition of histone deacetylase, including panobinostat, which show promise. ${ }^{7.8}$ Beyond these, there remains a challenge.

Most importantly, we face the dilemma in myeloma of 'stemness, ' a complicated entity reflecting the fact that, while CD38 targeting has been very successful, and BCMA has been very encouraging, these targetsare relativelydownstreamofthemolecularontogenyofmyelomadevelopment, andthestemness that precedes it remains a challenge. In that setting, true immune adjuvants, such as elotuzumab, which activate natural killer cells on the myeloma cell surface have shown promise. ${ }^{9}$ However, these 
do not address the fundamental complexity of the disease and the essence of stemness. The ability to deliver an agent that is highly targeted and deliver efficacy into core disease is a major frontier. After CD38-targeted treatment has failed, extramedullary disease has become an important new area of unmet medical need. ${ }^{10}$ In the relapsed/refractory setting, after monoclonal antibodies and other platform approaches have run out of steam, what do we have to offer the patient? In that regard, new strategies are urgently needed.

\section{Q. Could you tell us a little about melflufen and its mechanism of action?}

Melflufen is an important new agent in my view. It is a novel peptide conjugate, ultimately a melphalan-derived warhead (melflufen flufenamide) that rapidly delivers its payload directly into the tumor cell. The drug conjugate is highly lipophilic and therefore safe, and readily enters the bone marrow. In contrast, melphalan is lipophobic and requires dose escalation. ${ }^{11}$ The conjugate is also highly dependent on the action of aminopeptidases, which are enriched in myeloma cells. ${ }^{12}$ This means that when the conjugate enters the cell, it is cleaved and the active drug is enriched in the cell, where it shows striking and targeted cytotoxicity against the tumor. This confers several advantages, including an absence of dose-limiting toxicities such as mucositis, (which is dose-limiting for melphalan), and alopecia. In addition, its cytotoxicity is over 50 times higher than that of melphalan, allowing it to overcome resistance to prior chemotherapy exposure. ${ }^{13}$ We are also beginning to understand other properties of melflufen, including its strong antiangiogenic action, which is important since plasma cells are enriched with new vessel formation, and disrupting that process is crucial. This is also important in extramedullary disease, making it an additionally very attractive drug in the setting of relapsed/refractory $\mathrm{MM}$.

\section{Q. What was the rationale for and design of the 0-12-M1 study?}

The first-in-human trial of melflufen in solid tumors demonstrated that the conjugate could be safely administered at active doses; the primary toxicity was hematological. ${ }^{14}$ These findings informed the 0-12-M1 study, which was an international, multicenter study that had both dose escalation and dose expansion components, as a phase I/II study (ClinicalTrials.gov Identifier: NCT01897714). The study explored intravenous melflufen combined with dexamethasone for the majority of patients. ${ }^{5}$ In addition, a small cohort of patients received single-agent melflufen to demonstrate what the drug could achieve without the benefit of added steroids. It is important to bear in mind that, in patients with relapsed/refractory MM, everyone is steroid resistant. Despite this, the majority of novel agents benefit from the addition of steroids, and melflufen is no exception. In the phase II portion, we treated with the maximum tolerated dose that was defined from the phase I portion, and included the combination and single-agent cohorts. The study enrolled 75 patients over 7 sites: 23 in the phase I portion of the study and 58 in the phase II portion, of whom 13 were treated with single agent.

\section{Q. What were the major efficacy and safety findings of this study?}

The dose escalation study started at $15 \mathrm{mg}$, then $25 \mathrm{mg}$, and ultimately $40 \mathrm{mg}$, infused every 3 to 4 weeks, with the highest dose tested being $55 \mathrm{mg}$. Traditionally, we think of alkylating agents as being dosed per meter squared, but there is clear evidence that fixed dosing with alkylators can be effective. We therefore used fixed doses. At $15 \mathrm{mg}, 25 \mathrm{mg}$ and $40 \mathrm{mg}$, the drug was well tolerated. At $55 \mathrm{mg}$, neutropenia and thrombocytopenia occurred in three patients. We therefore did not test the highest planned dose of $70 \mathrm{mg}$. The maximum tolerated dose was defined at $40 \mathrm{mg}$. We also investigated the dosing schedule. Initially, we used a 3-weekly schedule, which was intensive and active, but we found that prolonging the interval to 4 weeks allowed more time for hematologic recovery, and so the 4-weekly schedule was adopted going forward. The dexamethasone dosage was $40 \mathrm{mg}$ once a week, serving as a convenient low dosage. ${ }^{5}$

When we looked at single-agent melflufen in the 11 patients who were available for evaluation of efficacy and the 13 who were evaluable for safety, $40 \mathrm{mg}$ was generally well tolerated and manageable in terms of side effects, but the response signal was modest. It was very clear that the dexamethasone was adding to the efficacy of the drug. The clinical benefit rate in the single-agent melflufen group was $23 \%$, whereas in the combination group it was $49 \%$. In addition, the partial response rate was $10 \%$ for the single-agent group and $31 \%$ in the combination group, suggesting that the combination should be used. In the 45 patients who received the combination, the clinical benefit rate was $50 \%$, which is encouraging in the setting of relapsed/refractory disease with an overall response rate of $31 \%$. Stable disease was achieved in a further $30 \%$. Overall, only $16 \%$ progressed; a favorable finding in this setting. In the time-to-event analyses, these responses were found to be quite durable, which is important as this study has considerable maturity in follow-up at a median of 28 months. Median progression-free survival (PFS) in all patients was 6 months and overall survival (OS) was 21 months. This provides evidence for activity in this population, with meaningful benefits in PFS and OS. ${ }^{5}$

The median number of prior therapies in the study population was 5 (range 4.0-6.5), reflecting a heavily pretreated population. A relatively small, but not trivial, number of patients had been exposed to antibodies (15 daratumumab and 2 elotuzumab), as opposed to the current treatment paradigm. Patients with high-risk cytogenetics were also well represented. ${ }^{5}$

In terms of safety, tolerability was favorable, and overall toxicities were mild to moderate and proved reversible. Cytopenia and neutropenia were the dominant toxicities, and proved manageable with dose reduction, dose delay and blood product support or growth factors. There were limited non-hematological toxicities; we did not see significant mucositis or alopecia. Gastrointestinal side effects were modest. The rate of serious adverse events was around $27 \%$, including pneumonia in four patients, and two septic events. The number of upper respiratory tract infections was quite low compared with other studies in this setting; however, there were infections in a minority of patients that contributed to mortality. There were four fatal events study-wide, but all in patients with rapidly progressing disease and within 30 days of the last dose of melflufen, with two of these infectious. The two septic events occurred immediately in patients who had been enrolled on the trial and had received one dose of melflufen; 5 significant bleeding did not occur.

\section{Q. What will be the next steps in the clinical development of melflufen?}

This study has defined that $40 \mathrm{mg}$ of melflufen and $40 \mathrm{mg}$ dexamethasone will be the platform going forward. It has also shown that this drug is effective in the setting of triple-class refractory patients. In terms of ongoing and future studies, the HORIZON study (ClinicalTrials.gov identifier: NCT02963493) is a large multicenter, international, phase ॥ trial that 
has enrolled over 150 patients; ${ }^{15}$ final results are promising and will be presented shortly. These findings confirm the efficacy that was seen in the 0-12-M1 study, and have also shown activity in extramedullary disease, a very important consideration as mentioned earlier. The OCEAN trial (ClinicalTrials.gov identifier: NCT03151811) is an international, randomized, phase III study comparing melflufen and dexamethasone with pomalidomide and dexamethasone in a less heavily pretreated group of patients (two or more prior lines of therapy), and is almost fully enrolled. ${ }^{16}$
Combination studies are also ongoing. The ANCHOR trial (ClinicalTrials.gov identifier: NCT03481556) is investigating the combination of melflufen and bortezomib in one cohort, and melflufen and daratumumab in another cohort. Early results have shown that combinations appear to be effective and generally well tolerated so far, suggesting that melflufen has the potential to be a partner drug with a number of existing drugs that are mainstays of MM therapy, and further enhance efficacy with the potential to meaningfully improve outcomes in patients with relapsed/refractory multiple myeloma. $\square$
1. National Cancer Institute. Surveillence, Epidemiology and End Results Program (SEER). Cancer Stat Facts: Myeloma. Available at: https://seer.cancer.gov/statfacts/html/mulmy.html laccessed April 27, 2020).

2. El-Amm J, Tabbara IA. Emerging therapies in multiple myeloma. Am I Clin Oncol. 2015:38:315-21.

3. Yong K, Delforge M, Driessen C, et al. Multiple myeloma: patient outcomes in real-world practice. Br J Haematol. 2016;175:252-64.

4. Kumar SK, Dimopoulos MA, Kastritis E, et al. Natural history of relapsed myeloma, refractory to immunomodulatory drugs and proteasome inhibitors: a multicenter IMWG study. Leukemia. 2017;31:2443-8.

5. Richardson PG, Bringhen $S$, Voorhees $P$, et al. Melflufen plus dexamethasone in relapsed and refractory multiple myeloma (O-12-M1): a multicentre, international, open-label, phase 1-2 study. Lancet Haematol. 2020;7:e395-407.

6. Bae J, Samur M, Richardson P, et al. Selective targeting of multiple myeloma by $\mathrm{B}$ cell maturation antigen (BCMA)-specific central memory CD8(+) cytotoxic T lymphocytes: immunotherapeutic application in vaccination and adoptive immunotherapy.
Leukemia. 2019;33:2208-26.

7. Chari A, Vogl DT, Gavriatopoulou M, et al. Oral selinexor-dexamethasone for triple-class refractory multiple myeloma. N Eng/ J Med. 2019;381:727-38.

8. Tzogani $K$, van Hennik $P$, Walsh I, et al. EMA review of panobinostat (Farydak) for the treatment of adult patients with relapsed and/or refractory multiple myeloma. Oncologist. 2018;23:631-6

9. Dimopoulos MA, Dytfeld D, Grosicki S, et al. Elotuzumab plus pomalidomide and dexamethasone for multiple myeloma. N Eng/ J Med. 2018;379:1811-22.

10. Rasche L, Weinhold N, Morgan GJ, et al. Immunologic approaches for the treatment of multiple myeloma. Cancer Treat Rev. 2017:55:190-9.

11. Wickstrom $M$, Nygren $P$, Larsson $R$, et al. Melflufen - a peptidase-potentiated alkylating agent in clinical trials. Oncotarget. 2017;8:66641-55.

12. Wickstrom $M$, Viktorsson $K$, Lundholm $L$, et al. The alkylating prodrug $\mathrm{J} 1 \mathrm{can}$ be activated by aminopeptidase $\mathrm{N}$, leading to possible target directed release of melphalan.
Biochem Pharmacol. 2010;79:1281-90.

13. Chauhan $\mathrm{D}$, Ray A, Viktorsson $\mathrm{K}$, et al. In vitro and in vivo antitumor activity of a novel alkylating agent, melphalan-flufenamide, against multiple myeloma cells. Clin Cancer Res. 2013:19:3019-31.

14. Berglund A, Ullen A, Lisyanskaya A, et al. First-in-human, phase I/lla clinical study of the peptidase potentiated alkylator melflufen administered every three weeks to patients with advanced solid tumor malignancies. Invest New Drugs. 2015;33:1232-41.

15. Richardson PG, Oriol A, Larocca A, et al. HORIZON (OP-106) study of melflufen in patients with relapsed/refractory multiple myeloma (RRMM) refractory to daratumumab and/or pomalidomide: updated efficacy and safety. Clin Lymphoma Myeloma LeuK. 2019;19(Suppl.1):S329-30.

16. Schjesvold F, Robak P, Pour $L$, et al. OCEAN: a randomized phase ॥ study of melflufen + dexamethasone to treat relapsed refractory multiple myeloma. Future Oncol. 2020;16:631-41. 